\title{
Evolvable Mars Campaign Long Duration Habitation Strategies: Architectural Approaches to Enable Human Exploration Missions
}

\author{
Matthew A. Simon \\ NASA Langley Research Center, Hampton, VA, 23681, USA \\ Larry Toups \\ NASA Johnson Space Center, Houston, TX, 77058 USA \\ A. Scott Howe \\ Jet Propulsion Laboratory, Pasadena, CA, 91109 USA \\ Samuel Wald \\ Georgia Tech, Atlanta, GA, 30332 USA
}

The Evolvable Mars Campaign (EMC) is the current NASA Mars mission planning effort which seeks to establish sustainable, realistic strategies to enable crewed Mars missions in the mid-2030s timeframe. The primary outcome of the Evolvable Mars Campaign is not to produce "The Plan" for sending humans to Mars, but instead its intent is to inform the Human Exploration and Operations Mission Directorate near-term key decisions and investment priorities to prepare for those types of missions. The FY'15 EMC effort focused upon analysis of integrated mission architectures to identify technically appealing transportation strategies, logistics build-up strategies, and vehicle designs for reaching and exploring Mars moons and Mars surface. As part of the development of this campaign, long duration habitats are required which are capable of supporting crew with limited resupply and crew abort during the Mars transit, Mars moons, and Mars surface segments of EMC missions. In particular, the EMC design team sought to design a single, affordable habitation system whose manufactured units could be outfitted uniquely for each of these missions and reused for multiple crewed missions. This habitat system must provide all of the functionality to safely support 4 crew for long durations while meeting mass and volume constraints for each of the mission segments set by the chosen transportation architecture and propulsion technologies.

This paper describes several proposed long-duration habitation strategies to enable the Evolvable Mars Campaign through improvements in mass, cost, and reusability, and presents results of analysis to compare the options and identify promising solutions. The concepts investigated include several monolithic concepts: monolithic clean sheet designs, and concepts which leverage the co-manifested payload capability of NASA's Space Launch System (SLS) to deliver habitable elements within the Universal Payload Adaptor between the SLS upper stage and the Orion/Service module on the top of the vehicle. Multiple modular habitat options for Mars surface and in-space missions are also considered with various functionality and volume splits between modules to find the best balance of reducing the single largest mass which must be delivered to a destination and reducing the number of separate elements which must be launched. Analysis results presented for each of these concepts in this paper include mass/volume/power sizing using parametric sizing tools, identification of unique operational constraints, and limited comments on the additional impacts of reusability/dormancy on system design. Finally, recommendations will be made for promising solutions which will be carried forward for consideration in the Evolvable Mars Campaign work. 


\section{Nomenclature}

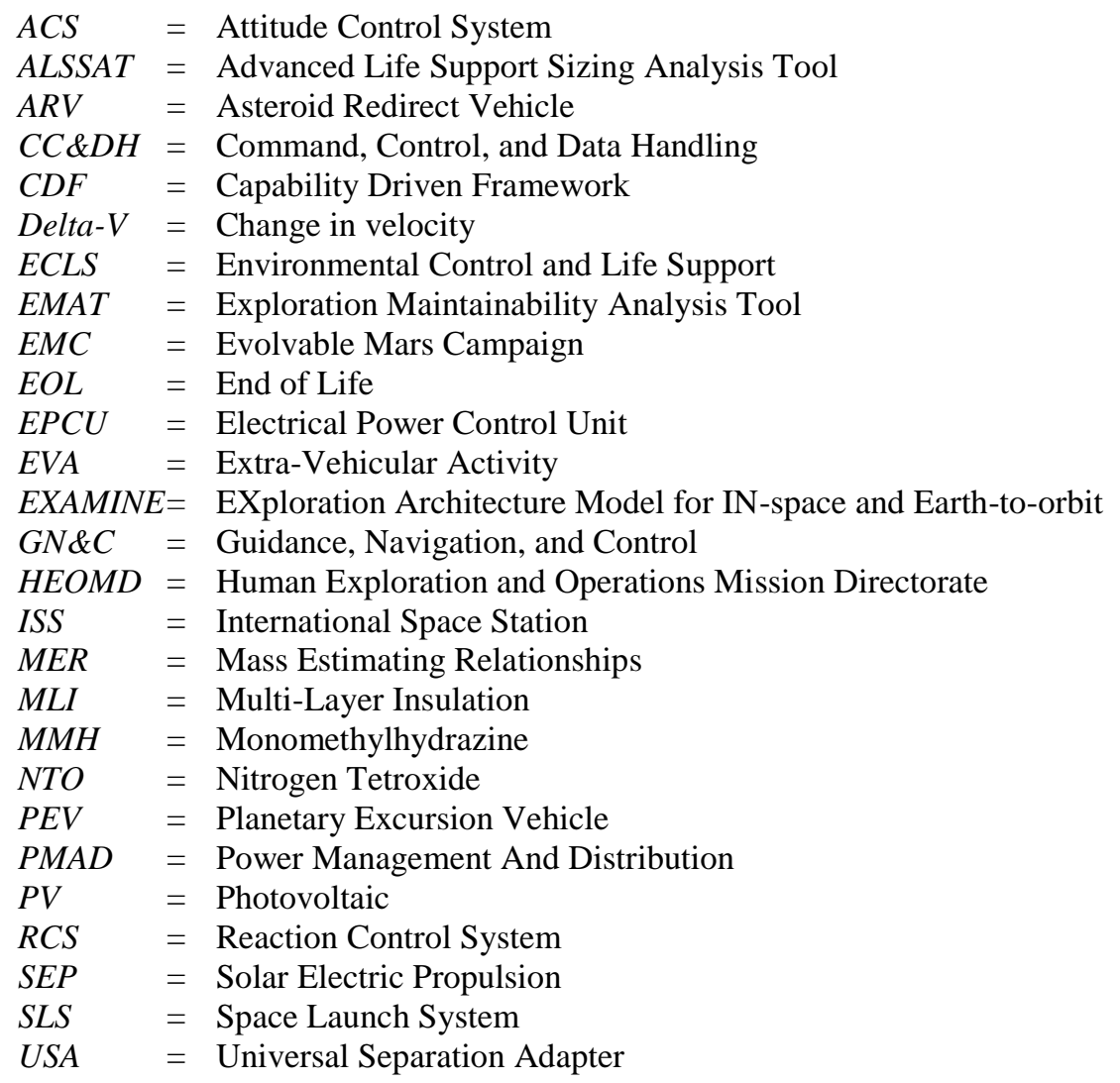

\section{Introduction}

$\mathrm{N}$ ASA is developing a "long-term strategy for achieving extended missions to Mars" with a goal of reaching the Mars vicinity with crew in the mid-2030s ${ }^{1}$. The Evolvable Mars Campaign ${ }^{1,2}$ (EMC) is an ongoing study investigating potential options for exploration missions to achieve this goal sustainably and affordably. This study is distinguished from other Mars mission planning efforts by its focus on reduction of the cost of Mars missions through the use of evolving near-term investments and reusable vehicles across a cadence of missions building up to Mars surface missions. It leverages a Capability-Driven Framework (CDF) strategy to phase development costs; incrementally and strategically developing capabilities in order to enable a continual cadence of progressively more challenging missions which build upon previous investments. For example, the development of low-thrust transportation stages and long-duration habitation capabilities enable a mission to deep space and Mars moons. The addition of capabilities to land and live on planetary surfaces enable additional missions such as a long duration Mars surface mission. This cadence of missions allows a gradual buildup of capabilities necessary for Mars surface missions over a longer period of time, reducing up-front spikes in the budget; improving the likelihood of Mars missions in a fiscally constrained environment. The EMC mission design efforts also reduce the cost of future Mars missions through the adoption of commonality and reusability on some of the elements including the low-thrust propulsion stages and short- and long- duration habitats. EMC mission concepts reuse a single transit vehicle and return to a single Mars surface landing site to reduce the number of production units which must be manufactured and launched. Additionally, the EMC leverages commonality in the design of habitation systems (both long and short duration) to eliminate some of the cost associated with new, unique designs. The FY' 15 EMC effort focused upon analysis of integrated mission architectures to identify technically appealing transportation strategies, logistics build-up strategies, and vehicle designs for reaching and exploring Mars moons and Mars surface.

Long-duration habitation for in-space and planetary surfaces represents a major capability within in the capability-driven Evolvable Mars Campaign. Habitats must be able to support four crewmembers for 300-1100 days in challenging environments with limited resupply, no crew abort, and long communication delays; all within 
constrained mass, volume, and power budgets ${ }^{2,3,4,5}$. In addition to these constraints, there are several characteristics of the EMC mission architecture which drive specific habitation design strategies. First, the design and manufacture of each new exploration element tend to be costly, so there is a desire within EMC to develop one common, longduration habitat system design (made up of one or multiple modules). These common modules share a common manufacturing infrastructure and outfitting can be used to build several versions with the appropriate modifications for transit, Mars moons, and Mars surface missions. Second, the current EMC in-space transportation and Mars landing performances place mass constraints on the habitat system. This makes large, monolithic habitat concepts (>40 metric tons) unwieldy for mission planning. For example, identified limitations of in-space delivery capabilities (specifically electric propulsion performance) have restricted Mars lander options to those capable of delivering payloads less than 18 metric tons (with options of up to 27 tons possible with additional investment in entry/landing technologies). This may require habitats to be delivered in multiple modules or as a single module which can be launched relatively empty and supplied with logistics and spares on the surface. Finally, there is a desire in the Evolvable Mars Campaign to reuse habitat systems for multiple missions with long periods of dormancy separating missions. This places additional requirements and design constraints on systems for refurbishment, repair, and recertification. Based upon these factors, there is a desire within the Evolvable Mars Campaign analysis work to investigate lightweight monolithic and modular habitat approaches and consider additional architectural/technological methods to further reduce the mass and improve reusability.

This paper describes various habitation strategies aimed at achieving these EMC goals. Section 2 describes the three habitats which are needed within the EMC, outlining the constraints and assumptions feeding current habitat designs. This section also outlines the methodology used to model these habitats to inform transportation and surface infrastructure analyses. Section 3 describes monolithic (single module) approaches including clean sheet designs and a concept using the cargo area within a stage adapter on crewed SLS flights as a habitable volume. Section 4 describes modular design concepts aimed at enabling smaller Mars landers. Finally Section 5 describes the implications of these findings and outline future work to refine habitat concepts.

\section{Long-Duration Habitat Design Assumptions and Constraints}

1. Habitat types and functional assumptions

There are three long-duration habitat systems in the Evolvable Mars Campaign:

1) a Mars moons habitat available in 2028 supporting crew for 300-550 days

2) a transit habitat available in 2032 supporting crew for up to 1100 days in deep space

3) a Mars surface habitat available in 2035 supporting crew for 300-550 days on the Martian surface

Each of these habitats support a crew of four, providing all required logistics and spares and varying amounts of power generation, power storage, and EVA functionality for each mission. Additionally, these habitats must keep crewmembers healthy and happy for all stages of increasingly challenging long duration missions with few opportunities for resupply or abort. These vehicles require hundreds of cubic meters of volume to accommodate support systems (including deep space communications, vehicle control, life support, etc.), crew activities (including meal preparation, exercise, sleeping quarters, etc.), EVA support, psychological acceptable layouts, and logistics access and storage. The habitats must also be designed to operate reliably for long durations with considerations like maintainability and supportability in mind. The full list of functional assumptions for long-duration EMC habitats are listed below:

Habitat Structure \& Mechanisms

- Metallic cylinder, Al-Al face sheet,- honeycomb structure

- $25 \mathrm{~m}^{3} /$ person habitable volume

- Secondary structure sized as $2.46 \mathrm{~kg} / \mathrm{m} 2$ of habitat structural area

- Integration structure $2 \%$ of habitat gross mass

- 4 x $0.5 \mathrm{~m}$ windows

- 1 exterior hatch for contingency EVAs

- 3 docking mechanisms -1 active, 2 passive

- Atmospheric Pressure $=101.3 \mathrm{kPa}$ (14.7 psia), $21 \%$ oxygen

Protection

- 20 layers Multi-Layer Insulation (MLI) covering external habitat surface for passive thermal control Power

- Habitat solar arrays provided when propulsion stage power is not sized to support the habitat 
- Electrical Power Control Unit (EPCU) $120 \mathrm{~V}$ dc Power Management and Distribution (PMAD) (92\% efficient)

- Variable numbers and sizes of Li-ion batteries to support transitions and eclipses

Environmental Control and Life Support (ECLS)

- Scaled ISS-level, partially closed ECLSS hardware for 380 days

- $+10 \%$ added life support mass for advanced maintenance and diagnostics

- 30 days open loop contingency consumables for critical subsystems (carbon dioxide removal, oxygen generation, water recovery)

- Includes fire detection and suppression

Crew Accommodations

- Food storage, individual crew quarters, sink(spigot), microwave oven, hand/mouth wash faucet, washer \& dryer, 2 vacuums, laptop, trash compactor, printer, hand tools \& accessories, test equipment, ergometer, photography equipment, exercise equipment, treadmill, table (microgravity)

Extra-Vehicular Activity (EVA)

- Assumed $6 \mathrm{~m}^{3}$ volume for internal contingency airlock (consistent with minimal airlock), 90\% EVA air reclamation

- Contingency EVAs at a rate of one 2-person EVA per month

Thermal Control

- External fluid loop for heat acquisition using ammonia

- Internal fluid loop for heat acquisition using $60 \%$ prop glycol/water

- Heat acquired from cabin \& avionics rejected using ISS-type radiators w/ 10 mil Ag-Teflon coating (parametrically determined from power loading)

Avionics

- Provide Command, Control, and Data Handling (CC\&DH), Guidance, Navigation, and Control (GN\&C) and Communications

Vehicle Control

- Provide $125 \mathrm{~m} / \mathrm{s}$ delta- $\mathrm{V}$ with nitrogen tetroxide/monomethylhydrazine (NTO/MMH) storable propellants when vehicle must perform maneuvers independent from attached propulsion stages

$\underline{\text { Utilization }}$

- $1000 \mathrm{~kg}$ unallocated utilization mass $+250 \mathrm{~kg}$ robotic assistant

$\underline{\text { Reserves }}$

- Margin growth Allocation - 20\% of basic mass

- Project Manager's Reserve - $10 \%$ of basic mass

In addition to these functional assumptions, a set of additional transportation system dimensions and mass constraints guide the design of the single, common habitat design which serves as the basis for the three longduration EMC habitats. This habitat design needs to be as mass efficient as possible as the power levels of the Asteroid Redirect Vehicle (ARV) ${ }^{6}$ Solar Electric Propulsion (SEP) systems being utilized in the architecture are at their limits for the transit habitat and delivery of the landers to Mars orbit. The habitats must be able to meet the mass constraints listed in Table 1 for the current transportation architecture (propulsive element designs and number of launches) to be feasible. Additionally, the habitats must also be able to be packaged with some other architectural elements within the currently assumed cargo SLS shroud. Meeting these constraints while providing the required functionality is a significant challenge. In particular, meeting the 18 ton Mars surface landing constraints currently being assumed by EMC has been a focus of recent habitation design efforts.

Table 1. Mass constraints for long-duration EMC habitats

\begin{tabular}{|l|l|}
\hline Vehicle & Mass Constraint \\
\hline Mars Transit Habitat & Gross mass $<40$ metric tons \\
\hline Mars Moons Habitat & $\begin{array}{l}\text { Total between Mars moons habitat, rover, and landing/mobility equipment } \\
\text { gross mass }<40 \text { metric tons }\end{array}$ \\
\hline Mars Surface Habitat & $\begin{array}{l}\text { Landed mass }<18 \text { or }<27 \text { metric tons (after offloading logistics and other } \\
\text { offloadable items.) }\end{array}$ \\
\hline
\end{tabular}


In order to create a functional habitat design which meets these constraints, several technologies and design features are assumed. In particular, the following advances are needed to enable EMC architectures:

- Lightweight structures

- Reliable, component level maintainable life support equipment with ISS level closure

- Some combination of low mass countermeasures, pharmaceuticals, crew selection, and revised risk posture to enable low mass for galactic cosmic ray radiation

- Advances to reduce spares mass

- Low mass exercise equipment $(<1000 \mathrm{~kg})$ occupying $<2 \mathrm{~m}^{3}$ volume when stowed

- Low mass behavioral health countermeasures

- Low mass microgravity physiological countermeasures

- High data rate forward link communications

- Communications delay-tolerant equipment and operations

- Autonomous vehicle systems monitoring and management

Additional advances which are being investigated include repurposing of expended logistics, in-situ manufacturing of spares, robotic assistants, and inflatable structures applications.

\section{Habitat Modeling}

In order to investigate various habitat design strategies which can 1) meet these constraints and 2) perform favorably in the current EMC transportation architectures, a parametric habitat design trade study is conducted utilizing a parametric sizing tool based upon EXAMINE (EXploration Architecture Model for IN-space and Earthto-orbit) ${ }^{7}$. EXAMINE is an architecture modeling framework developed at NASA LaRC which contains a collection of parametric performance and sizing tools and algorithms that enable users to model a various types of architectural elements. Originating from a collection of existing NASA spacecraft sizing toolsets, it includes and expands upon JSC's Envision? ${ }^{7}$ MSFC's MER (Mass Estimating Relationships) database, JSC's ALSSAT (Advanced Life Support Sizing Analysis Tool), and a dynamic spares sizing tool EMAT (Exploration Maintainability Analysis Tool) ${ }^{8}$. EXAMINE provides detailed architecture element-specific sizing in mass, volume, and power for Levels 1, 2, and (occasionally) 3 detail. It also provides a framework for performing an integrated sizing analysis across all elements in an architecture concept, which enables trades studies to improve DRM and element designs.

Inputs are varied in an instantiation of the EXAMINE model created to parametrically size conceptual habitation elements. This habitat sizing model is then used to investigate multiple habitation strategies to find the right balance between several factors influencing the EMC transportation architecture. First, a balance must be struck between vehicle mass and the cost of technologies required to achieve those masses. Architectural and systems-level integration solutions which require only strategic investment in a few technologies will be preferred over multiple technology-only solutions achieving similar mass savings. Additionally, habitats should emphasize mission architecture simplicity which is characterized by reduced numbers of launches, landers, vehicle rendezvous, and infrastructure setup operations. This simplicity greatly reduces overall mission cost and risk while increasing feasibility of the architecture by requiring the development of fewer elements. Finally, options which leverage existing developments greatly reduce development cost, normally at the expense of more mass. While clean-sheet options optimize mass, they represent a brand new element design. Existing or commercially available elements can potentially provide cost savings, though an in-depth cost analysis is necessary to validate anticipated benefits.

There are two primary approaches investigated in the EMC for long duration habitats: monolithic and modular. Monolithic indicates that all of the functions required to support the crew during the mission are located within a single module. Modular approaches rely on the aggregation of multiple modules to provide all of these functions. The following sections detail some options for each and comment upon their potential benefits and weaknesses over alternate approaches. As there are multiple transportation architectures being considered within the EMC, all concepts shown in this paper are associated with the Hybrid SEP-chemical architecture detailed in Reference 4

\section{Monolithic Habitat Design Options}

\section{A. Clean-Sheet Monolithic Concepts}

Clean-sheet monolithic concepts are the most basic of the habitat design strategies. They feature a custom-sized habitat sized to include all the required functionality and volume to support crew within one module. Figure 1 shows an example of an EMC monolithic habitat sized for an 1100 day Mars transit and Table 2 shows the logistics loading 
for the Hybrid Sep-Chemical architecture over the proposed opportunities. The benefit of clean sheet monolithic is its simplicity and relatively low mass for the total habitation system. Since modular concepts must add hatches, bulkheads, and other redundant spacecraft systems, they are inherently more massive for a similar overall volume when compared to monolithic concepts. The major disadvantage of the monolithic habitat strategy is the inability to lower the delivered mass of the large habitat beyond a certain threshold.
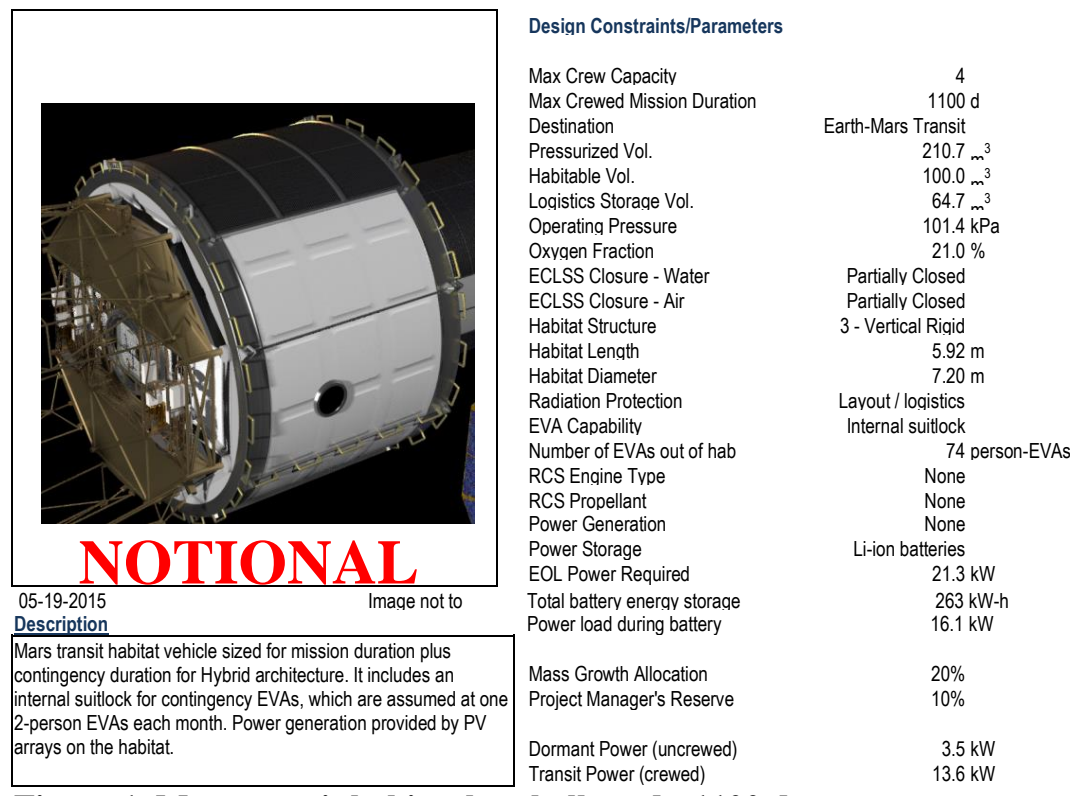

\begin{tabular}{|l|r|}
\hline Category & Mass, $\mathbf{~ k g}$ \\
\hline Structure & $\mathbf{5 , 1 8 5}$ \\
\hline Protection & 268 \\
\hline Propulsion & 0 \\
\hline Power & 1,980 \\
\hline Control (ACS/RCS) & 0 \\
\hline Avionics & 453 \\
\hline ECLSS & 3,507 \\
\hline EVA systems & 2,425 \\
\hline Thermal Control System & 1,115 \\
\hline Crew Equipment & 1,749 \\
\hline Utilization & 1,000 \\
\hline Growth & $\mathbf{5 , 1 7 2}$ \\
\hline DRY MASS SUBTOTAL & $\mathbf{2 2 , 8 5 4}$ \\
\hline Logistics including ECLSS Consumables & See Table 2 \\
\hline Reserve and Residual Prop. & $\mathbf{0}$ \\
\hline INERT MASS SUBTOTAL & $\mathbf{x x x}$ \\
\hline Propellant & $\mathbf{0}$ \\
\hline TOTAL WET MASS & $\mathbf{x x x}$ \\
\hline & \\
& \\
& \\
& \\
& \\
& \\
\end{tabular}

Figure 1. Mars transit habitat baseball card - 1100 days

Table 2. Logistics loading of habitat for various opportunities

\begin{tabular}{|c|}
\hline Habitat Dry Mass (kg.) \\
\hline 22,854 \\
\hline
\end{tabular}

\begin{tabular}{|c|c|c|c|c|}
\hline \multicolumn{5}{|c|}{ Total Logistics Payload In DSH at Burn (kg.) } \\
\hline Opportunity & TMI & MOI & TEI & EOI \\
\hline 2033 & 17,404 & 14,774 & 9,887 & 7,206 \\
\hline 2039 & 17,691 & 14,217 & 10,589 & 7,511 \\
\hline 2043 & 17,691 & 14,465 & 10,852 & 7,526 \\
\hline
\end{tabular}

$=$

\begin{tabular}{|c|c|c|c|c|}
\hline \multicolumn{6}{|c|}{ Total Hybrid Transit Habitat Inert Mass at Burn (kg.) } \\
\hline Opportunity & TMI & MOI & TEI & EOI \\
\hline $\mathbf{2 0 3 3}$ & 40,258 & 37,628 & 32,741 & 30,060 \\
\hline $\mathbf{2 0 3 9}$ & 40,545 & 37,071 & 33,443 & 30,365 \\
\hline $\mathbf{2 0 4 3}$ & 40,545 & 37,319 & 33,706 & 30,380 \\
\hline
\end{tabular}


For example, the mass breakdown shown at the right hand side of Figure 1 shows a habitat dry mass of $\sim 23$ tons. The dry mass of Mars surface and Mars moons habitats is similarly 21-23 tons, with variations due to outfitting. These masses do include a few items that would be offloadable, but ideally this would represent the indivisible delivered mass of the transit habitat if all logistics were delivered separately. As $\sim 22$ tons is more than the desired 18 tons for small Mars surface landers, this dry mass is problematic. An offloading analysis performed to address this issue shows that removing additional offloadable components can reduce the Mars surface habitat mass down to a minimum of $\sim 17$ tons which would enable the habitat to be delivered on an $\sim 18$ ton lander with approximately 30 days of logistics for crew. However, the amount of offloaded components greatly increases the complexity of the setup operations and somewhat increases the risk to crew by requiring critical rendezvous on the surface with logistics modules. Additionally, this places the loaded mass of the habitats right against the $\sim 18$ ton threshold, which makes it susceptible to becoming infeasible when/if habitat mass grows. This additional complexity and lack of margin are the main challenges of implementing a monolithic surface habitat on a $\sim 18$ ton surface lander.

There are a few ongoing design efforts refining habitat designs to determine if monolithic surface habitat approaches can meet the 18 ton constraint without over-complicating the surface/landing operations. Additional investigations in EMC are currently underway to determine if developing a more capable lander somewhere between 18 and 27 tons can enable a more desirable architecture with fewer total launches/landers.

\section{B. SLS Universal Stage Adapter - Derived Monolithic Concepts}

Another approach being investigated in the EMC is geared at allowing habitat elements to be launched upon crewed SLS launches to fully utilize the available launches to aggregate exploration elements. Several configurations have been proposed for a SLS Universal Separation Adapter (USA) that will be used between the Exploration Upper Stage (EUS) and the Orion Crew Exploration Vehicle (CEV). Studies were conducted to determine whether habitation elements could be carried within the USA volume, as possible secondary payloads in an Orion launch. It is assumed that the USA structure would be used as-is, with added pressurized bulkheads to create a pressurized cabin volume for habitation elements. Additionally, hatches would need to be added to allow for transfer in and out of the pressurized volume. In effect, the USA volume could allow a habitat to be brought up in the "trunk" of the launch vehicle, where the Orion SEV is the primary payload. Figure 2 shows an example of adding a habitation element into a notional USA concept.

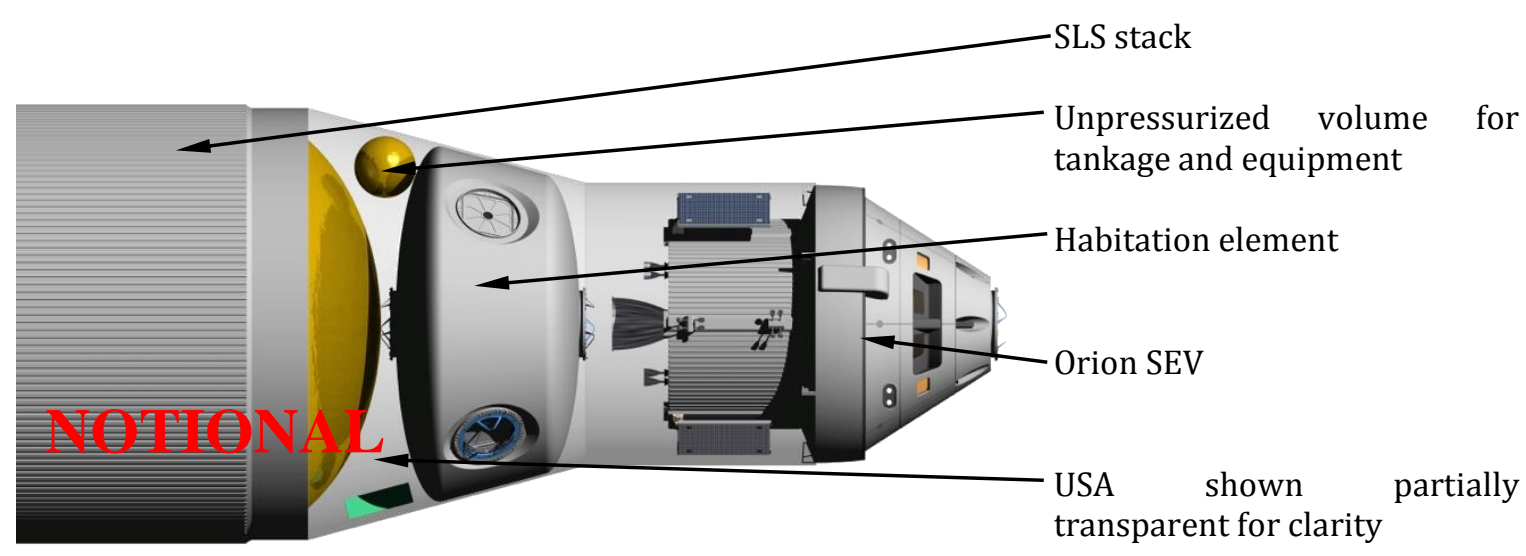

Figure 2. Habitation elements carried in a notional SLS Universal Separation Adapter (USA)

Several options were studied with the USA-derived "trunk" habitats that would allow the Orion SEV to separate from the stack, dock with the habitat, provide for inflatable habitat volume extension, protect EVA porches, and provide additional vehicle docking ports (Figure 3). The results indicated that some versions of the "trunk" habitat concepts are feasible from an operational perspective. 


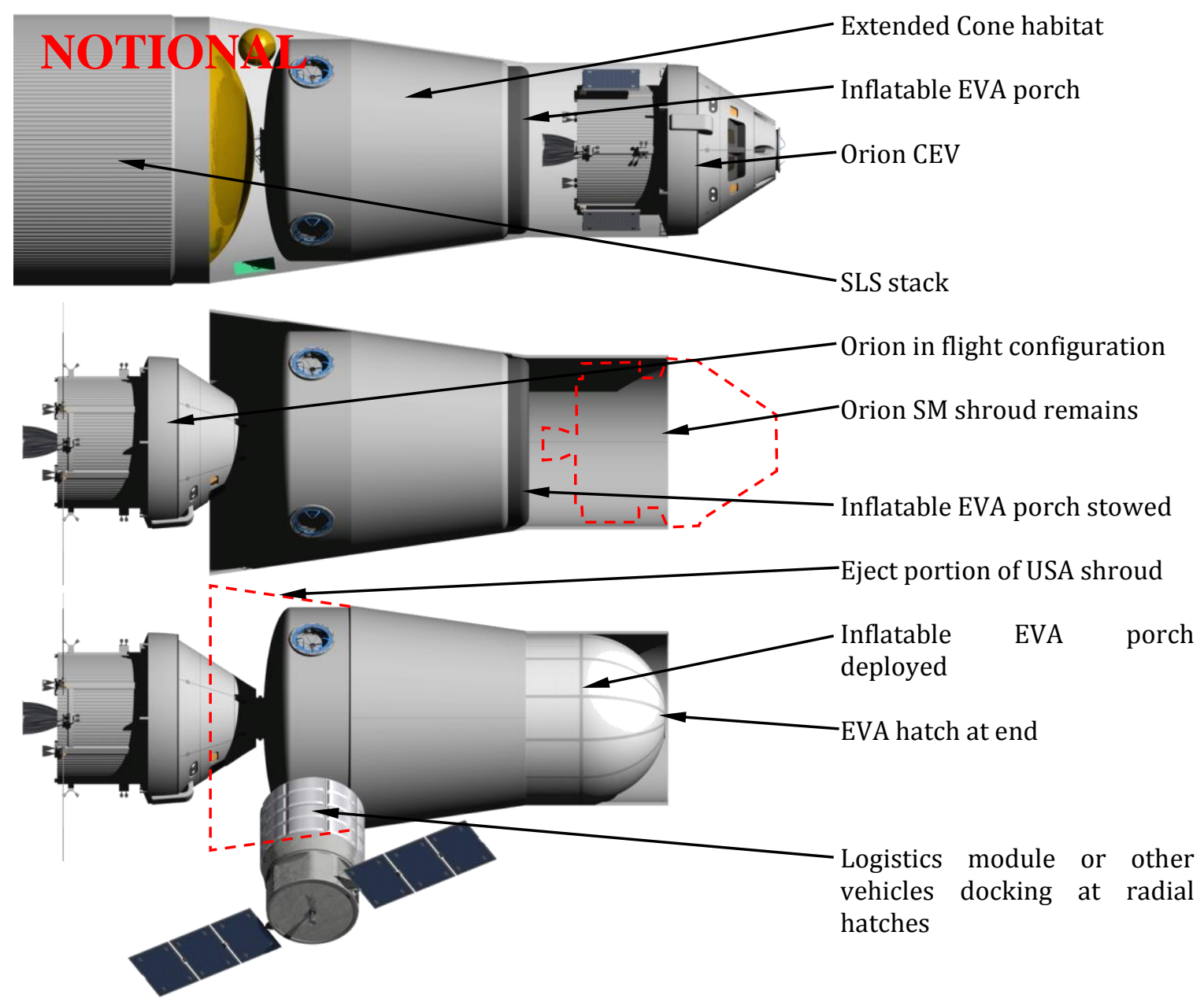

Figure 3. Configuration options for a notional SLS / USA-derived "trunk" habitat

It was assumed that the potential payload capacity for SLS 1B USAs would be $\sim 10$ tons and SLS 2 USAs would be $\sim 15$ tons. A study was performed to understand the available volumes which could be created in various USA geometries and what mass would be available for outfitting of the interior after accounting for the additional structure required to make an USA pressurizable. Masses for the habitation elements were constrained by the capacity of the launch vehicle beyond the USA and Orion payload masses. Volumes varied, and were constrained by the configuration of the various USA options. The results of initial studies are shown in Table 3. Several of the options enable pressurized volumes on par with those carried in the clean-sheet monolithic similar to the concept shown in Figure 1. Additionally, combined USA and habitat structure masses of 3-6 tons, leaves 4 to 7 tons (in the 10 ton payload limit) and 9 to 12 tons (in the 15 ton payload limit) for built in outfitting. Based upon the estimated masses of difficult to offload subsystems like life support, thermal, and power, it seems preliminarily feasible that an USA-derived habitat concept could be developed. 
Table 3. SLS / USA-derived "trunk" habitation element masses and volumes

\begin{tabular}{|l|c|c|c|c|c|c|c|} 
Option & $\begin{array}{c}\text { Habitat } \\
\text { Volume } \\
\text { (m3) }\end{array}$ & $\begin{array}{c}\text { Inflatable } \\
\text { Volume } \\
\text { (m3) }\end{array}$ & \multicolumn{1}{c}{$\begin{array}{c}\text { Total } \\
\text { Pressurized } \\
\text { Vol. (m3) }\end{array}$} & $\begin{array}{c}\text { USA } \\
\text { Mass } \\
\text { (kg) }\end{array}$ & $\begin{array}{c}\text { Habitat } \\
\text { Mass } \\
\text { (kg) }\end{array}$ & $\begin{array}{c}\text { Inflatable } \\
\text { Mass } \\
\text { (kg) }\end{array}$ & $\begin{array}{c}\text { Total } \\
\text { Mass } \\
\text { (kg) }\end{array}$ \\
\hline Short Stack & 95 & & 95 & 2,500 & 686 & & 3,186 \\
\hline $\begin{array}{l}\text { Short Stack } \\
\text { Inflatable }\end{array}$ & 95 & 248 & 343 & 2,500 & 686 & 652 & 3,838 \\
\hline Cone Cylinder & 327 & & 327 & 5,200 & 762 & & 5,962 \\
\hline Cylinder Cone & 175 & & 175 & 3,800 & 1,645 & & 5,445 \\
\hline Extended Cone & 260 & & 260 & 5,200 & 711 & & 5,900 \\
\hline
\end{tabular}

However, despite being feasible, the potential limitations of USA-derived habitat concepts are numerous. It does open up the possibility of commercial flights for some of the outfitting flights, but additional analysis is needed to prove that utilizing this strategy will actually reduce cost, as there is no guarantee it will reduce the total number of launches. This concept is also sensitive to launch vehicle mass growth. The 10 to 15 ton payload capacity of the SLS may disappear as additional engineering and manufacturing issues arise in the development of the SLS. Additionally, this USA-derived habitat concept is the only driver for a larger USA. Launch vehicle designers desire to minimize the mass of this section of the launch vehicle and currently plan to stage the structure during the ascent. As it is not clear that this habitat concept is a significant enabler of more effective mission architectures, it is unlikely that the USA geometry will be protected for its possible development. Ongoing efforts are not currently planned for these concepts, but may be reintroduced at a later time.

In summary, monolithic options are feasible, but face significant challenges for Mars surface. One option to correct this is to enforce commonality between transit and Mars moons habitat, but exclude commonality from a Mars surface perspective by designing a customized Mars surface habitat capable of meeting lander constraints. Another option is to simply increase lander capabilities to enable a common design between all three Mars mission habitats. Finally, the final option is to enforce commonality through the three habitats with the existing lander constraints by splitting them up to reduce the largest single piece which must be landed on the Martian surface. This does enforce some additional constraints on surface mobility and lander packaging manifests, but shows promise for achieving leaner missions with reduced landings. The next section describes some ongoing efforts to investigate the potential benefit of modular habitats in the context of the EMC missions.

\section{Modular Habitat Design Options}

\section{A. Clean-Sheet Monolithic Concepts}

Modular habitat systems have the potential to provide a wide range of benefits to a mission or campaign architecture. The modular habitat architecture is motivated by mutual interdependencies with other elements and systems. Moving from a single monolithic structure to multiple smaller habitat modules can help to simplify the operations required move the elements both on the Martian surface and in microgravity. It also reduces the launch and lander payload requirements by reducing the mass and size of the largest elements, and allowing for alternative lander packaging manifests. In addition, modular schemes may also reduce the complexity of resupply operations by allowing new logistics to be added without requiring the crew to transport tons of cargo between modules for assembly and storage. Like monolithic approaches, opportunities for commonality of pressure shells and other systems are also enabled by some modular architectures, which can in turn help to reduce manufacturing costs. Additional pressure volumes may also serve to reduce risk to the crew due to fire and decompression, allowing them a safe haven from which to stage and plan a solution to the issue.

However, modular habitation strategies may also bring various downsides and penalties ${ }^{9}$. Mass penalties are generally associated with duplicate systems necessary for each module, such as hatches, fire suppression, lighting, and air distribution and contaminant control. Structural mass penalties are also incurred due to the lower volume to mass efficiency of smaller radius modules, assuming constant length. Packaging efficiency for launch and landing may also be decreased if the space between individual cylindrical habitat modules cannot be filled by other payloads. Finally, modular concepts drive the design of surface offloading and mobility systems. The requirement for the currently assumed surface offloading and mobility considerations if delivery of modular components less than 10 s ton apiece. Delivery of larger modular pieces will require update of the surface system architecture. Despite 
these downsides, the EMC team is interested in evaluating how modular habitation options could impact the entire system across the campaign, and has begun to look at a few of the potential modular habitat options.

The EMC habitation team has set out a few modular options for analysis in order to trade them with previously described monolithic options. These modular options provide the same habitat functionality as the monolithic habitats split across multiple modules. Two of these concepts are listed below, and are also shown in Figure 4.

- Concept A -

- Surface: One large primary habitable and functional volume, with separate logistics modules under 10 tons to allow for use of mobility systems to move and mate modules

- Concept B -

- Moons: Common primary module, larger 500 day logistics module.

- Transit: Common primary module, two 500 day logistics modules.

- Surface: One large primary habitable and functional volume with mass of 18 tons, with separate logistics and offloadable item modules under 10 tons to allow for use of common 18 ton lander across EMC.

- Moons: Common primary module, larger 500 day logistics module.

- Transit: Common primary module, two 500 day logistics modules.

Note that the "larger 500 day" logistics modules may be split up to enforce small habitat commonality with surface logistics. Preliminary analysis has shown that these modular options can fit within the current EMC architecture and may provide more flexibility to the design of other campaign elements.

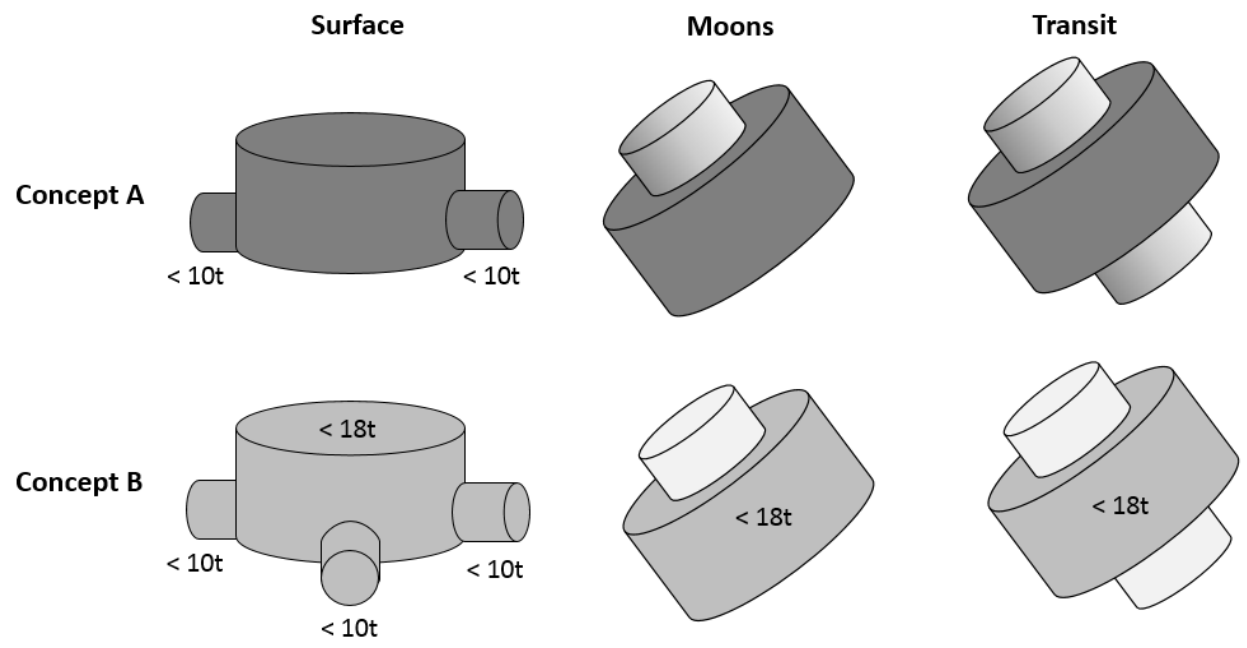

Figure 4. Clean-sheet modular habitation concepts

Concept B, comprising of one large primary habitation module and multiple smaller logistics modules, allows for the use of the smallest lander concept of 18 tons, and enables easy movement and rendezvous of the logistics modules with the larger module. Sizing of this habitat system was completed using the same methods and assumptions as those of the monolithic concepts. A summary of the surface habitat module masses and volumes, as well as a comparison can be seen in Table 4. This concept requires three common logistics modules in order to contain all of the necessary pressurized logistics as well as the offloaded items that must be reassembled by the crew in the main module upon their arrival. The empty mass penalty for this modular habitat is about $50 \%$, which is significant if evaluating concepts on habitat mass alone. However, more work is necessary in order to evaluate the overall campaign-wide impact of these concepts. Lander, propulsion, destination operations, and aggregation teams will all incorporate this concept and others into their trade space in order to fully understand their implications. 
Table 4. Concept B modular habitation system masses and comparison with monolithic habitat system

\begin{tabular}{lrrrr}
\hline Module & $\begin{array}{c}\text { Empty Mass } \\
(\mathbf{k g})\end{array}$ & $\begin{array}{c}\text { Outfitted Mass } \\
(\mathbf{k g})\end{array}$ & $\begin{array}{c}\text { Habitable } \\
\text { Volume } \\
(\mathbf{m 3})\end{array}$ & $\begin{array}{c}\text { Pressurized } \\
\text { Volume } \\
(\mathbf{m 3})\end{array}$ \\
\hline Primary & 18,003 & 18,003 & 100.00 & 150.66 \\
\hline Logistics & 4,035 & 9,438 & 8.33 & 33.30 \\
\hline Logistics & 4,035 & 9,438 & 8.33 & 33.30 \\
\hline Logistics & 4,035 & 9,438 & 8.33 & 33.30 \\
\hline Total & $\mathbf{3 3 , 5 5 7}$ & $\mathbf{4 6 , 3 1 8}$ & $\mathbf{1 2 4 . 9 8}$ & $\mathbf{2 5 0 . 5 7}$ \\
\hline Monolithic & 22,492 & 34,531 & 100.00 & 183.59 \\
\hline \% Delta & $\mathbf{+ 4 9 \%}$ & $\mathbf{+ 3 4 \%}$ & $\mathbf{+ 2 5 \%}$ & $\mathbf{+ 3 6 \%}$ \\
\hline
\end{tabular}

\section{B. Evolvable Modular Habitat Concepts.}

All of the habitat concepts investigated thus far in this paper propose new investments to create a workable habitation system. An alternative approach is to identify several habitable elements that will be available by the first long-duration habitat launch planned in 2028 that could be assembled into a working, long-duration habitation system with reduced investment. Additionally, it is anticipated that an initial cislunar habitation system will be needed in the early 2020s to provide short duration (30-60 days) habitation to prove out long-duration systems and operations. If a habitat system could be developed which addressed this need as part of its development, the resulting evolvable strategy could greatly reduce required investments by NASA for some microgravity applications.

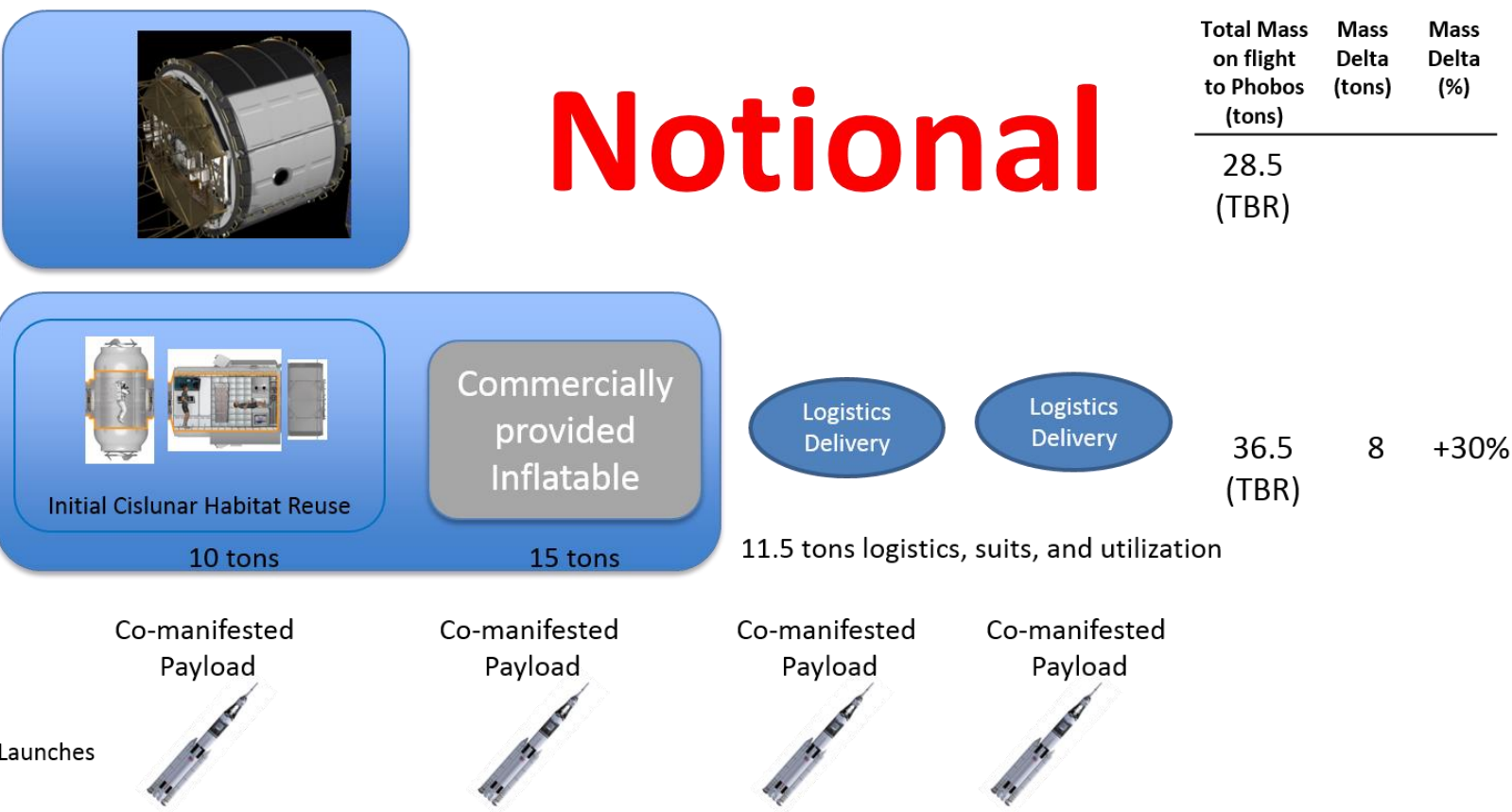

Figure 5. Evolvable modular habitat concept

An evolvable modular habitat concept is currently being investigated at a pre-conceptual level for feasibility. In this concept, commercially producible elements can be aggregated during SLS crewed launches utilizing the comanifested payload capability (10 tons before 2028, 15 tons after 2028). An initial cis-lunar habitat can be constructed from a Planetary Excursion Vehicle cabin - common airlock and a commercially available logistics module and service module as shown in Figure 5. This vehicle can be outfitted for 30 day missions under 10 tons. By adding a commercially producible inflatable habitat near $180 \mathrm{~m}^{3}$ which is less than 15 tons when partially

11

American Institute of Aeronautics and Astronautics 
outfitted, the total net habitable volume necessary to support a long duration mission is provided similar to that of the monolithic concept shown in Figure 1. This resulting habitat system can be 'topped off' in two logistics flights and perform the Mars moons mission, while being aggregated through co-manifested or commercial deliveries. Similar concepts could also be created for a Mars transit habitat, but Mars surface is anticipated to be too mass constrained for this approach to be feasible.

Initial concepts have shown that an evolvable habitat concept is feasible under the aforementioned constraint, but there are several drawbacks to such an approach. First, the 30\% increase in mass at trans-Mars injection burn, has performance impacts on the transportation system, particularly for the transit habitat case which is already near its 40 ton limit. Second, the necessity of 4 launches for a single mission is considerably more complicated than the simpler monolithic options. There may be additional issues with long aggregation times on perishable consumables. Ongoing studies will continue to search for an optimal evolvable modular habitation system which performs well in the context of the EMC, while the transportation team assesses how the transportation architecture might be adjusted to enable this potentially lower cost approach.

\section{Conclusion}

One important consideration which has not been previously mentioned is the importance of how reusability and long periods of dormant operations will affect habitat design and the selection of the most advantageous habitation strategy. The EMC is built upon the premise of reusability, but ongoing work is currently seeking to quantify the impacts to subsystem designs and masses. It is anticipated that autonomous vehicle health maintenance systems and substantially modified logistics supplies will be necessary, but the issue of how spares and routine maintenance of subsystems will be handled is a frequent topic of current team discussion. In addition, long periods of dormancy are anticipated to be an issue for perishable goods and systems with limited expected lifetimes. Information and procedures for interrupted occupancy of the International Space Station are being investigated to develop a plan for these challenges and will be released in the near future.

In summary, several habitation approaches have been described in the previous section. The current baseline habitat concept being carried in the EMC analysis is the clean-sheet monolithic approach, but the trades described above are leading the EMC analysis teams to investigate alternate mission architectures while concurrently developing higher fidelity parametric tools to adequately capture the difference between proposed concepts independent of large modeling uncertainties. In particular, the EMC team's near term focus is to understand how the modular concepts change the transportation architecture.

\section{References}

${ }^{1}$ Craig, D. A., Herrmann, N. B., and Troutman, P. A. "The Evolvable Mars Campaign - Study Status," IEEE Aerospace Conference 2015, Big Sky, MT, 2015.

${ }^{2}$ Craig, D., Herrmann, N., and Troutman, P., "Pioneering Space Through the Evolvable Mars Campaign," 2015 AIAA Space Conference, Pasadena, CA, 2015.

${ }^{3}$ Percy, T., McGuire, M., and Polsgrove, T., "In-space Transportation for NASA's Evolvable Mars Campaign," 2015 AIAA Space Conference, Pasadena, CA, 2015.

${ }^{4}$ Merrill, R., Chai, P., Jones, C., Komar, D., and Qu, M., "An Integrated Hybrid Transportation Architecture for Human Mars Expeditions,” 2015 AIAA Space Conference, Pasadena, CA, 2015.

${ }^{5}$ Goodliff, K., Cirillo, W., Mattfeld, B., Stromgren, C., and Shyface, H., "Comparison of Human Exploration Architecture and Campaign Approaches," 2015 AIAA Space Conference, Pasadena, CA, 2015.

${ }^{6}$ Mazanek, D.D., et al, "Asteroid Redirect Robotic Mission: Robotic Boulder Capture Option Overview," 2014 AIAA Space Conference, San Diego, CA, 2014.

${ }^{6}$ Komar, D. R., Hoffman, J., and Olds, A., "Framework for the Parametric System Modeling of Space Exploration. Architectures," AIAA-2008-7845, 2008.

${ }^{8}$ Stromgren, C., Terry, M., Cirillo, W., Goodliff, K. and Maxwell, A., "Design and Application of the Exploration Maintainability Analysis Tool," AIAA Space 2012 Conference and Exposition, AIAA-2012-5323, Pasadena, CA, 2012.

${ }^{9}$ Simon, M.A., Toups, L., Smitherman, D., "Potential Applications of Modularity to Enable a Deep Space Habitation Capability for Future Human Exploration Beyond Low-Earth Orbit," GLEX-2012.05.3.6 x12574, 2012 Global Space Exploration Conference, Washington, D.C., 2012. 